\title{
LHC constraints on 3-3-1 models
}

\author{
Camilo Salazar, ${ }^{a}$ Richard H. Benavides, ${ }^{c}$ William A. Ponce ${ }^{a}$ and Eduardo Rojas ${ }^{a, b}$ \\ ${ }^{a}$ Instituto de Física, Universidad de Antioquia, \\ Calle 70 No. 52-21, Medellín, Colombia \\ ${ }^{b}$ Laboratorio de Física Teórica e Computação Científica, Universidade Cruzeiro do Sul, \\ 01506-000, São Paulo, Brazil \\ ${ }^{c}$ Facultad de Ciencias Exactas y Aplicadas, Instituto Tecnológico Metropolitano, \\ Calle 73 No 76 A - 354, Vía el Volador, Medellín, Colombia \\ E-mail: camilo@gfif.udea.edu.co, richardbenavides@itm.edu.co, \\ wponce@fisica.udea.edu.co, eduardo.rojas@cruzeirodosul.edu.br
}

ABSTRACT: The ATLAS detector data on di-lepton production is used in order to impose constraints on $Z^{\prime}$ boson masses associated with a variety of 3-3-1 and $E_{6}$ motivated $Z^{\prime}$ models. Lower mass bounds for the different models are established at $95 \%$ confidence level. Our numerical analysis is extrapolated up to $14 \mathrm{TeV}$, and further to $30 \mathrm{TeV}$ and $100 \mathrm{TeV}$, for a broad range of luminosities. Some of our results can be compared with the ATLAS published bounds, being, for those cases, in fairly good agreement. We also report the vector and axial charges for all the 3-3-1-motivated $Z^{\prime}$ models without exotic electric charges for leptons, known in the literature. To the best of our knowledge most of this charges were not reported before.

KeYwords: Hadronic Colliders

ARXiv EPrint: 1503.03519 


\section{Contents}

1 Introduction 1

$2 \mathrm{SU}(3)_{c} \times \mathrm{SU}(3)_{L} \times \mathrm{U}(1)_{x}$ models 3

2.1 The minimal model 4

2.2 3-3-1 models without exotic electric charges 4

$\begin{array}{lll}3 & \text { Statistical analysis and results } & 6\end{array}$

$\begin{array}{llr}4 & \text { Conclusions } & 9\end{array}$

$\begin{array}{ll}\text { A Differential cross-section } & 11\end{array}$

B The 3-3-1 couplings 12

\section{Introduction}

The existence of new neutral vector bosons $Z^{\prime}$ beyond the one associated with the $\mathrm{SU}(3)_{c} \otimes$ $\mathrm{SU}(2)_{L} \otimes \mathrm{U}(1)_{Y}$ local gauge group of the Standard Model $(\mathrm{SM})^{1}$ [1], is a clear prediction of new physics, related to extra $\mathrm{U}(1)$ factors appearing in every regular chain of the breaking of larger gauge groups down to the SM one [2].

A systematic study of additional $\mathrm{U}(1)$ symmetries is possible just by restricting to the study of the lowest dimensional representations of larger gauge groups and their branching rules [3]. As it is well known, a family non universal $Z^{\prime}$ coupling leads to Flavor-Changing Neutral Currents (FCNC), and possibly to new CP-violating effects [2]. To avoid these inconveniences, some of the first models with physics beyond the SM incorporate the assumption of family universality, condition quite restrictive to such an extent that, it is not possible to construct a minimal extension of the SM just by adding a U(1) factor to the SM local gauge group, without the introduction of new fermion fields [4-6]; that is, it is not possible a $Z^{\prime}$ interaction just with the current content of the particles in the SM.

The requirement of universality for the $\mathrm{U}(1)$ charges and, in consequence anomaly cancellation in every family, leads in a natural way into $E_{6}$ subgroups in most of the cases. As a gauge group, $E_{6}$ is the only exceptional group with complex representations that is anomaly free in all its representations [7]. Some $E_{6}$ subgroups, such as the original unification groups $\mathrm{SU}(5), \mathrm{SO}(10)$, and the Left-Right symmetric models $\mathrm{SU}(4) \times \mathrm{SU}(2)_{L} \times$ $\mathrm{SU}(2)_{R}$ with their corresponding supersymmetric realizations, are between the most widely known extensions of the SM. For a classification of $\mathrm{U}(1)^{\prime}$ symmetries contained in $E_{6}$ see references $[8,9]$.

\footnotetext{
${ }^{1}$ For an excellent compendium of the SM, see ref. [1].
} 
Early in the nineties, some work pointed out to the conclusion that universality must not be taken for granted for models with physics beyond the SM. In particular, under some suitable assumptions, many non universal models were able to evade the FCNC constraints. Following this trend of ideas, the $\mathrm{SU}(3)_{c} \otimes \mathrm{SU}(3)_{L} \otimes \mathrm{U}(1)_{x}$ models (3-3-1 for short) were proposed by allowing anomaly cancellation between fermions in different families [10-18].

For the most popular 3-3-1 models $[11,14,16]$, three families is the simplest possible choice of matter content in order to have anomaly cancellation. So, one of the most appealing features of those models is to provide explanation for the family replication problem (also known as the generation number problem), which is a long standing issue in particle physics; furthermore, they provide some indications of why the top family is the heaviest one [19]. Also, 3-3-1 models are among the most interesting new physics scenarios with new sources of CP and flavor violation [20], making them the most suitable ones for flavor studies [21-28].

The first 3-3-1 model for three families was sketched originally in ref. [10], where references to previous $\mathrm{SU}(3) \otimes \mathrm{U}(1)$ models for one and two families can be found. Then, in refs. $[11,12]$ the so called minimal version of the model was introduced, minimal in the sense that it does not contain lepton fields beyond the ones present in the SM. Next, came the 3-3-1 family model with right handed neutrinos, rediscovered in refs. [13-15] (the first 3-3-1 family model with right handed neutrinos was introduced in ref. [10]). The three family model with exotic electrons was introduced in the literature in ref. [16], a classification of 3-3-1 models without exotic electric charges was done in refs. [17]; and finally, the so called economical 3-3-1 model appeared in ref. [18].

Since the gauge group for the 3-3-1 models is not simple, neither semi-simple, there is not a neat prediction of the electroweak mixing angle, neither there is an explanation for the quantization of the electric charge using only the cancellation of anomalies (the quantum constraints); ${ }^{2}$ but, as in the SM, the inclusion of the classical constraints leads in a simple way to the quantization of the electric charge [29], conclusion linked to the generation number problem in ref. [30]. As a last remark, it has been shown that the most general Yukawa couplings in some 3-3-1 models, include in a natural way a Peccei-Quinn type symmetry that can be extended to the entire Lagrangian in a very elegant way [31], and by using appropriate extra fields, the resulting axion can be made invisible.

In the eventual discovery of a new neutral vector boson, it will be important the experimental determination of its coupling to the standard model fermions. However, the discrimination between the possible $Z^{\prime}$ models could be challenging at the LHC, owing to the reduced number of high resolution channels in hadron colliders. So, in order to carry out the statistical analysis, it is necessary to combine the LHC data with electroweak precision data. For 3-3-1 models, the most important constraints come from the flavor changing neutral currents (FCNC); in consequence, it is important to establish the models for which the LHC and/or the FCNC constraints are dominant; that is, which kind of constraints exclude a wider region in the parameter space. It is also important to set the range of

\footnotetext{
${ }^{2}$ In grand unified theories with simple gauge groups, the electric charge is quantized because the charge operator is a linear combination of generators of the unifying group.
} 
parameters and models for which the LHC and the FCNC constraints are comparable to each other; in such a case, it is convenient to combine both.

In order to set the present $95 \%$ confidence level (CL) limits and the projected ones, we follow closely the CDF methods explained in ref. [32]. For the exact expression of the $\chi^{2}$ function, the theoretical formulas of the SM expected values, and the statistical analysis, we follow the work of the authors in refs. [9, 33]. As an improvement, we update the program used in [9] with the set CTQ10 of parton distribution functions [34] which allow us to reach higher energies than previous releases.

In this paper we present the $Z^{\prime}$ charges for all the 3-3-1 models Without Exotic Electric Charges for leptons, known in the literature, most of them new results. Then, using the recent dilepton data reported by ATLAS in reference [35] we calculate the lower bounds for $M_{Z^{\prime}}$ at $95 \% \mathrm{CL}$, and project also at $95 \% \mathrm{CL}$ for the $\mathrm{LHC}$ and $\mathrm{VLHC}^{3}$ forthcoming energies and luminosities.

The paper is organized as follows: in section 2 we review the different 3-3-1 models present in the literature; in section 3 we derive the present 95\% CL limits and the projected ones on the $Z^{\prime}$ mass for typical LHC energies and luminosities. The section 4 summarizes our conclusions. Technical appendixes at the end present the differential cross-section formulas used in the analysis and the charges of the SM fermions for the different 3-3-1 models Without exotic electric charges for leptons, in the literature.

\section{$2 \mathrm{SU}(3)_{c} \times \mathrm{SU}(3)_{L} \times \mathrm{U}(1)_{x}$ models}

The different models based on a 3-3-1 gauge symmetry are classified according to the electric charge operator which is given by

$$
Q=a \lambda_{3}+\frac{1}{\sqrt{3}} b \lambda_{8}+x I_{3}
$$

where $\lambda_{\alpha}, \alpha=1,2, \ldots, 8$ are the Gell-Mann matrices for $\mathrm{SU}(3)_{L}$ normalized as $\operatorname{Tr}\left(\lambda_{\alpha} \lambda_{\beta}\right)=$ $2 \delta_{\alpha \beta}$ and $I_{3}=D g(1,1,1)$ is the diagonal $3 \times 3$ unit matrix. $a=1 / 2$ if one assumes that the isospin $\mathrm{SU}(2)_{L}$ of the $\mathrm{SM}$ is entirely embedded in $\mathrm{SU}(3)_{L}$ and $b$ is a free parameter which defines the different possible models. The $x$ values must be obtained by anomaly cancellation.

The covariant derivative for the electroweak sector is given now by:

$$
D_{\mu}=\partial_{\mu}-i \frac{g}{2} \sum_{\alpha=1}^{8} \lambda_{\alpha} A_{\mu}^{\alpha}-i g_{1} x X_{\mu} I_{3}
$$

where $A_{\mu}^{\alpha}$ and $X_{\mu}$ are the gauge fields of $\mathrm{SU}(3)_{L}$ and $\mathrm{U}(1)_{x}$ respectively, and $g$ and $g_{1}$ are the coupling constants of the same gauge structures.

\footnotetext{
${ }^{3}$ VLHC stands for Very Large Hadron Collider that would accelerate protons to energies of about $100 \mathrm{TeV}[36,37]$.
} 
$x=0$ for $A_{\mu}^{\alpha}$, the 8 gauge fields of $\mathrm{SU}(3)_{L}$, and thus eq. (2.1) implies:

$$
\sum_{\alpha} \lambda_{\alpha} A_{\mu}^{\alpha}=\sqrt{2}\left(\begin{array}{ccc}
D_{1 \mu}^{0} & W_{\mu}^{+} & K_{\mu}^{(b+1 / 2)} \\
W_{\mu}^{-} & D_{2 \mu}^{0} & K_{\mu}^{(b-1 / 2)} \\
K_{\mu}^{-(b+1 / 2)} & K_{\mu}^{-(b-1 / 2)} & D_{3 \mu}^{0}
\end{array}\right),
$$

where $W_{\mu}^{ \pm}=\left(A_{\mu}^{1} \pm i A_{\mu}^{2}\right) / \sqrt{2}, \quad K_{\mu}^{ \pm(b+1 / 2)}=\left(A_{\mu}^{4} \pm i A_{\mu}^{5}\right) / \sqrt{2}, \quad K_{\mu}^{ \pm(b-1 / 2)}=\left(A_{\mu}^{6} \pm\right.$ $\left.i A_{\mu}^{7}\right) / \sqrt{2}, \quad D_{1 \mu}^{0}=A_{\mu}^{3} / \sqrt{2}+A_{\mu}^{8} / \sqrt{6}, \quad D_{2 \mu}^{0}=-A_{\mu}^{3} / \sqrt{2}+A_{\mu}^{8} / \sqrt{6}$, and $D_{3 \mu}^{0}=-2 A_{\mu}^{8} / \sqrt{6}$. The upper index on the gauge bosons stand for the electric charge of the particles, some of them being functions of the $b$ parameter.

In this paper we consider all the 3-3-1 models which do not include leptons with exotic electric charges; they correspond to the $b$ parameter in equation (2.1) equal only to $\pm 1 / 2$ and $3 / 2$. Recently, $b$ has been used as a free parameter for doing FCNC phenomenology in the context if 3-3-1 model [22, 24, 38, 39]; in some of those papers [22], fermion and gauge bosons structures have been constructed for arbitrary $b$ values, in particular, field structures for $b= \pm 1 / 2, \pm 1,3 / 2$ are considered (for $b \pm 1$, gauge and lepton fields with half integer electric charges are present).

\section{$2.1 \quad$ The minimal model}

In refs. $[11,12,19,40-42]$ it was shown that, for $b=3 / 2$ in eq. (2.1), the following fermion structure is free of all the gauge anomalies:

$$
\psi_{l L}^{T}=\left(l^{-}, \nu_{l}^{0}, l^{+}\right)_{L} \sim\left(1,3^{*}, 0\right), Q_{i L}^{T}=\left(u_{i}, d_{i}, X_{i}\right)_{L} \sim(3,3,-1 / 3), Q_{3 L}^{T}=\left(d_{3}, u_{3}, Y\right) \sim
$$
$\left(3,3^{*}, 2 / 3\right)$, where $l=e, \mu, \tau$ is a family lepton index, $i=1,2$ for the first two quark families, and the numbers after the similarity sign means 3-3-1 representations. The right handed fields are $u_{a L}^{c} \sim\left(3^{*}, 1,-2 / 3\right), d_{a L}^{c} \sim\left(3^{*}, 1,1 / 3\right), X_{i L}^{c} \sim\left(3^{*}, 1,4 / 3\right)$ and $Y_{L}^{c} \sim\left(3^{*}, 1,-5 / 3\right)$, where $a=1,2,3$ is the quark family index and there are three exotic quarks, two with electric charge $-4 / 3\left(X_{i}\right)$ and other with electric charge $5 / 3(Y)$. This version is called minimal in the literature, because its lepton content is just the one present in the SM.

\section{$2.2 \quad 3-3-1$ models without exotic electric charges}

If one wishes to avoid exotic electric charges as the ones present for the new quarks in the minimal model, one must choose $b= \pm 1 / 2$, in eq. (2.1). Following [18] we start with the following six sets of fermions which are closed in the sense that they contain the antiparticles of the charged particles:

- $S_{1}=\left[\left(\nu_{\alpha}^{0}, \alpha^{-}, E_{\alpha}^{-}\right) ; \alpha^{+} ; E_{\alpha}^{+}\right]_{L}$ with quantum numbers $(1,3,-2 / 3) ;(1,1,1)$ and $(1,1,1)$ respectively.

- $S_{2}=\left[\left(\alpha^{-}, \nu_{\alpha}, N_{\alpha}^{0}\right) ; \alpha^{+}\right]_{L}$ with quantum numbers $\left(1,3^{*},-1 / 3\right)$ and $(1,1,1)$ respectively.

- $S_{3}=\left[(d, u, U) ; u^{c} ; d^{c} ; U^{c}\right]_{L}$ with quantum numbers $\left(3,3^{*}, 1 / 3\right) ;\left(3^{*}, 1,-2 / 3\right)\left(3^{*}, 1,1 / 3\right)$ and $\left(3^{*}, 1,-2 / 3\right)$ respectively. 
- $S_{4}=\left[(u, d, D) ; u^{c} ; d^{c} ; D^{c}\right]_{L}$ with quantum numbers $(3,3,0) ;\left(3^{*}, 1,-2 / 3\right) ;\left(3^{*}, 1,1 / 3\right)$ and $\left(3^{*}, 1,1 / 3\right)$ respectively.

- $S_{5}=\left[\left(e^{-}, \nu_{e}, N_{1}^{0}\right) ;\left(E^{-}, N_{2}^{0}, N_{3}^{0}\right) ;\left(N_{4}^{0}, E^{+}, e^{+}\right)\right]_{L}$ with quantum numbers $\left(1,3^{*},-1 / 3\right)$; $\left(1,3^{*},-1 / 3\right)$ and $\left(1,3^{*}, 2 / 3\right)$ respectively.

- $S_{6}=\left[\left(\nu_{e}, e^{-}, E_{1}^{-}\right) ;\left(E_{2}^{+}, N_{1}^{0}, N_{2}^{0}\right) ;\left(N_{3}^{0}, E_{2}^{-}, E_{3}^{-}\right) ; e^{+} ; E_{1}^{+} ; E_{3}^{+}\right]_{L}$ with quantum numbers $(1,3,-2 / 3) ;(1,3,1 / 3) ;(1,3,-2 / 3) ;(111),(111)$; and (111) respectively.

The different anomalies for these six sets are [18] found in table 1. With this table, anomalyfree models, without exotic electric charges can be constructed for one, two or more families. As noted in ref. [18], there are eight three-family models that are anomaly free, which are:

- Model A: named in the literature "model with right-handed neutrinos". Its fermion structure is given by $3 S_{2}+S_{3}+2 S_{4}$. This model was introduced for first time in the literature in ref. [10], rediscovered in refs. [13-15], with the weak charges presented in ref. [43].

- Model B: named in the literature "Model with exotic electrons". This model was introduced in the literature in ref. [16] and its lepton sector was studied in ref. [44]. Its fermion structure is given by $3 S_{1}+2 S_{3}+S_{4}$.

- Model C: named in the literature "model with unique lepton generation one" (three different lepton families). Introduced for the first time in ref. [17] and its was partially analyzed in ref. [45], where the weak charges only for the leptons were calculated. Its fermion structure is given by $S_{1}+S_{2}+S_{3}+2 S_{4}+S_{5}$.

- Model D: named in the literature "model with unique lepton generation two". Introduced for the first time in ref. [17] and it was partially analyzed in ref. [45], where the weak charges only for the leptons were calculated. Its fermion structure is given by $S_{1}+S_{2}+2 S_{3}+S_{4}+S_{6}$.

- Model E: we name it as "model hybrid one" (two different lepton structures). Its fermion structure is given by $S_{3}+2 S_{4}+2 S_{5}+S_{6}$.

- Model F: we name it as "model hybrid two". Its fermion structure is given by $2 S_{3}+S_{4}+S_{5}+2 S_{6}$.

- Model G: we name it as "carbon copy one" (three identical families as in the SM). The fermion structure is the same as the representation of the $\mathbf{2 7}$ of the $E_{6}$ group i.e., $3\left(S_{4}+S_{5}\right)$. The fermion weak charges were presented in the literature in ref. [46]

- Model H: we name it as "carbon copy two". The fermion weak charges for this model were presented in the literature in ref. [47]. Its fermion structure is given by $3\left(S_{3}+S_{6}\right)$. 


\begin{tabular}{|l|cccccc|}
\hline Anomalies & $S_{1}$ & $S_{2}$ & $S_{3}$ & $S_{4}$ & $S_{5}$ & $S_{6}$ \\
\hline$\left[\mathrm{SU}(3)_{C}\right]^{2} \mathrm{U}(1)_{x}$ & 0 & 0 & 0 & 0 & 0 & 0 \\
{$\left[\mathrm{SU}(3)_{L}\right]^{2} \mathrm{U}(1)_{x}$} & $-2 / 3$ & $-1 / 3$ & 1 & 0 & 0 & -1 \\
{$[\mathrm{Grav}]^{2} \mathrm{U}(1)_{x}$} & 0 & 0 & 0 & 0 & 0 & 0 \\
{$\left[\mathrm{U}(1)_{x}\right]^{3}$} & $10 / 9$ & $8 / 9$ & $-12 / 9$ & $-6 / 9$ & $6 / 9$ & $12 / 9$ \\
{$\left[\mathrm{SU}(3)_{L}\right]^{3}$} & 1 & -1 & -3 & 3 & -3 & 3 \\
\hline
\end{tabular}

Table 1. Anomalies for 3-3-1 fermion fields structures.

\section{Statistical analysis and results}

In reference [35] the ATLAS detector at the Large Hadron Collider was used to search for high-mass resonances decaying to dielectron or dimuon final states. The experiment analyze proton-proton collisions at a center of mass energy of $8 \mathrm{TeV}$ and a integrated luminosity of $20.3 \mathrm{fb}^{-1}$ in the dielectron channel and $20.5 \mathrm{fb}^{-1}$ in the dimuon channel. From this data they report 95\% CL upper limits on the total cross-section of $Z^{\prime}$ decaying to dilepton final states in $p p$ collisions. In the aforementioned work the ATLAS collaboration reported limits for $Z_{\chi}$ and $Z_{\psi}$, which are $E_{6}$-motivated $Z^{\prime}$ models, and for the Sequential Standard Model (SSM) $Z_{\text {SSM }}^{\prime}$, which is a model with couplings to the SM fermions identical to the $Z$. Part of the purpose of this work is to extend this analysis to 3-3-1 models and also to the remaining $E_{6}$ models which were not considered by ATLAS. In this vein we also carry out our own statistical analysis by using a binned likelihood function. The likelihood function is defined as the product of the Poisson probabilities over all the dilepton invariant mass bins, i.e.,

$$
L(\vec{n} \mid \vec{\mu}) \equiv \prod \frac{e^{-\mu_{i}} \mu_{i}^{n_{i}}}{n_{i} !} .
$$

The confidence levels limits correspond to contours of constant Log-Likelihood Ratio $\operatorname{LLR}\left(M_{Z^{\prime}}\right)$, with

$$
\operatorname{LLR}\left(M_{Z^{\prime}}\right)=-2 \log \frac{L\left(\vec{n} \mid \vec{\mu}^{\prime}\right)}{L(\vec{n} \mid \vec{\mu})}=2 \sum_{i}\left(\mu_{i}^{\prime}-\mu_{i}+n_{i} \ln \frac{\mu_{i}}{\mu_{i}^{\prime}}\right),
$$

where $n_{i}$ is the observed number of events in every bin, $\mu_{i}$ and $\mu_{i}^{\prime}$ are the expected number of events in every bin for the SM and the SM extended by a $Z^{\prime}$ respectively. The explicit expression for the expected number of events is given by

$$
\mu_{i}=K_{i} \int_{\text {bin }} \frac{d \sigma^{\mathrm{NLO}}}{d M_{l^{+} l^{-}}},
$$

where $K_{i}$ stand for all the correction factors necessary to get the expected number of events in every bin. This corrections include final state radiation corrections, dilepton invariant mass resolution effects, NNLO QCD, acceptance and efficiency correction factors. We got the $K_{i}$ from the ratio between the published SM values for $\mu_{i}$ from figure 2 in [35] over the NLO cross-section in the SM, $\sigma^{\mathrm{NLO}}$ from eq. (A.1), in every bin. In the calculation of the expected number of events we only took into account the couplings of the $Z^{\prime}$ to the SM 
fermions. In order to find the $95 \% \mathrm{CL}$ limits on the masses for $E_{6}$-motivated $Z^{\prime}$ models we fix the $Z^{\prime}$ coupling strength to $g_{2}=0.4615$ (see eq. (B.1) for the $g_{2}$ definition) and $g_{2}=0.7433$ for $3-3-1$ models and the sequential standard model $Z_{\mathrm{SSM}}$. In our calculation we fix to zero the mixing angle between the $Z$ and the $Z^{\prime}$ in agreement with the most recent constraints [56-59]. It is important to notice that despite the fact that the number of observed events in every bin is Poisson distributed, according with the Wilks's theorem the minimum of the likelihood ratio as a function of the $Z^{\prime}$ mass, follows a $\chi^{2}$ distribution with degrees of freedom equal to the difference of the number of parameters between the two models $^{4}$ [60]. So, the one-parameter 95\% CL limits correspond to LLR-LLR ${ }_{\min }=3.84$, where $L_{L R}$ min is the minimum of the LLR as a function of the $Z^{\prime}$ mass. For this analysis we used the thirty five high-invariant-mass bins for which the statistical errors are dominant, we did not include low-invariant-mass bins because other uncertainties become important. ${ }^{5}$ Following ATLAS, the bin width is constant in $\log M_{l^{+} l^{-}}$; i.e., the border between two adjacent bins, $M_{l^{+} l^{-}}^{i}$, is given by an exponential function $M_{l^{+} l^{-}}^{i}=M_{l^{+} l^{-}}^{1} \exp [(i-1) \times$ constant], where $M_{l^{+} l^{-}}^{1}$ is the leftmost invariant mass value and $i=1,2, \cdots$. We fit the ATLAS invariant mass coordinates to this functional form, getting a good agreement.

The results of this analysis are shown in table 2 and table 3 where the $95 \%$ CL lower mass limits for some 3-3-1 models and various $E_{6}$-motivated $Z^{\prime}$ models are shown. The second and third columns contain the $95 \%$ CL lower mass limits obtained from the dimuon and dielectron data. In our analysis the lower bounds for the $Z^{\prime}$ mass of the SSM are $2.57 \mathrm{TeV}$ in the dimuon channel and $2.80 \mathrm{TeV}$ in the dielectron channel, which are in good agreement with the quoted limits by ATLAS for this model i.e., $2.53 \mathrm{TeV}$ in the dimuon channel and $2.79 \mathrm{TeV}$ in the dielectron channel. In the fourth column appears the 95\% CL lower mass limits for the combined channels. In order to combine the dielectron and dimuon data we add the respective LLR, neglecting systematic uncertainties and correlations. The validity of this procedure only depends on the validity of the results. As we can see in table 3 for the models $Z_{\chi}, Z_{\psi}$ and $Z_{\mathrm{SSM}}$ we obtain $2.66 \mathrm{TeV}, 2.51 \mathrm{TeV}$ and $2.92 \mathrm{TeV}$ which differs at most $1.5 \%$ with the corresponding ATLAS results $2.62 \mathrm{TeV}, 2.51 \mathrm{TeV}$ and $2.90 \mathrm{TeV}$ respectively.

In order to make a cross-check of our analysis we make an alternative calculation of the lower bounds. As can be seen from figure 5 in ref. [35], for narrow width resonances the 95\% CL upper limits on the total cross-section of signal events is almost model independent for $Z^{\prime}$ masses below $2 \mathrm{TeV}$. For larger masses, the constraints are model dependent. Since ATLAS does not report upper limits for all the models, a useful approximation in the $2-3 \mathrm{TeV}$ range is to read the $Z^{\prime}$ mass lower limit at the intersection of the theoretical total cross-section ${ }^{6} \sigma^{\mathrm{NLO}}\left(p p \rightarrow Z^{\prime} \rightarrow l^{+} l^{-}\right)$eq. (A.1) with the $95 \%$ CL upper limit on the total cross-section of a narrow width resonance. Here, we use the upper limit on the total cross-section of the $Z_{\mathrm{SSM}}$ model in figure 5 of [35] which was the usual choice in earlier literature (see for example [61]). This approximation allows to estimate the $95 \%$ CL lower mass limits differing from the corresponding LHC ones in at most a few percent as can be seen in the fifth column in table 3.

\footnotetext{
${ }^{4}$ Provided that certain regularity conditions are met.

${ }^{5}$ For example at low-invariant-mass the theoretical uncertainties become larger than the statistical ones.

${ }^{6}$ In order to obtain $\sigma^{\mathrm{NLO}}$ it is necessary to integrate eq. (A.1).
} 


\begin{tabular}{|l|cccc|}
\hline$Z^{\prime}$ & $\mu^{-} \mu^{+}$ & $e^{-} e^{+}$ & $l^{-} l^{+}$ & intersection \\
\hline$Z_{331 \mathrm{~A}}$ & 2.36 & 2.48 & 2.65 & 2.60 \\
$Z_{331 \mathrm{~B}}$ & 2.66 & 2.72 & 2.89 & 2.88 \\
$Z_{331 \mathrm{C}}$ & 2.34 & 2.45 & 2.57 & 2.59 \\
$Z_{331 \mathrm{D}}$ & 2.68 & 2.73 & 2.91 & 2.91 \\
$Z_{331 \mathrm{E}}$ & 2.71 & 2.71 & 2.89 & 2.87 \\
$Z_{331 \mathrm{~F}}$ & 2.67 & 2.73 & 2.90 & 2.88 \\
$Z_{331 \mathrm{G}}$ & 2.74 & 2.71 & 2.92 & 2.91 \\
$Z_{331 \mathrm{H}}$ & 2.65 & 2.71 & 2.88 & 2.87 \\
$Z_{331 \text { minimal }}$ & 2.68 & 2.65 & 2.94 & 2.93 \\
\hline
\end{tabular}

Table 2. 95\% CL lower mass limits (in TeV) for some 3-3-1 $Z^{\prime}$ models. The second and third columns contain the $95 \%$ CL lower mass limits obtained from the dimuon and dielectron data in [35] respectively (see the text for details). In the fourth column appears the 95\% CL lower mass limits for the combined dielectron and dimuon channels. Given in the fifth column are the lower mass limits obtained by finding the intersection of the total cross-section $\sigma^{\mathrm{NLO}}$ eq. (A.1) with the ATLAS 95\% CL upper limit on the total cross-section of the $Z_{\mathrm{SSM}}$.

\begin{tabular}{|l|ccccc|}
\hline$Z^{\prime}$ & $\mu^{-} \mu^{+}$ & $e^{-} e^{+}$ & $l^{-} l^{+}$ & intersection & ATLAS \\
\hline$Z_{\chi}[8]$ & 2.42 & 2.48 & 2.66 & 2.59 & 2.62 \\
$Z_{\psi}[8]$ & 2.20 & 2.35 & 2.51 & 2.42 & 2.51 \\
$Z_{\eta}[48]$ & 2.31 & 2.38 & 2.56 & 2.47 & - \\
$Z_{L R}[49-51]$ & 2.44 & 2.54 & 2.68 & 2.71 & - \\
$Z_{R}[8]$ & 2.56 & 2.68 & 2.87 & 2.80 & - \\
$Z_{N}[52,53]$ & 2.20 & 2.36 & 2.51 & 2.44 & - \\
$Z_{S}[54,55]$ & 2.36 & 2.42 & 2.54 & 2.53 & - \\
$Z_{I}[8]$ & 2.31 & 2.37 & 2.52 & 2.48 & - \\
$Z_{B-L}[49]$ & 2.57 & 2.68 & 2.84 & 2.81 & - \\
$Z_{\not \supset}[9]$ & 2.75 & 2.84 & 2.97 & 2.94 & - \\
$Z_{\mathrm{SSM}}$ & 2.57 & 2.80 & 2.92 & 2.91 & 2.90 \\
\hline
\end{tabular}

Table 3. $95 \% \mathrm{CL}$ lower mass limits (in TeV) for various $E_{6}$-motivated $Z^{\prime}$ models and the SSM. The second and third columns contain the 95\% CL lower mass limits obtained from the dimuon and dielectron data in [35] respectively (see the text for details). In the fourth column appears the $95 \%$ CL lower mass limits for the combined dielectron and dimuon channels. Given in the fifth column are the lower mass limits obtained by finding the intersection of the total cross-section $\sigma^{N L O}$ eq. (A.1) with the ATLAS $95 \%$ CL upper limit on the total cross-section of the $Z_{\mathrm{SSM}}$. In the sixth column are the ATLAS published constraints on the respective model.

In figure 1 the projected 95\% CL exclusion limits on $M_{Z^{\prime}}$ for several 3-3-1 models are shown. We obtain this limits by assuming that the number of observed events $n_{i}$ is equal to the SM expectation $\mu_{i}$ in every bin. In order to obtain the bin size for every center of mass energy and luminosity in figure 1, we took ten high-invariant-mass bins and varied the bin size until the mass limit reaches a maximum. To obtain the limits listed there we use 30 bins. We also have assumed for the product acceptancexefficiency the ATLAS result for 


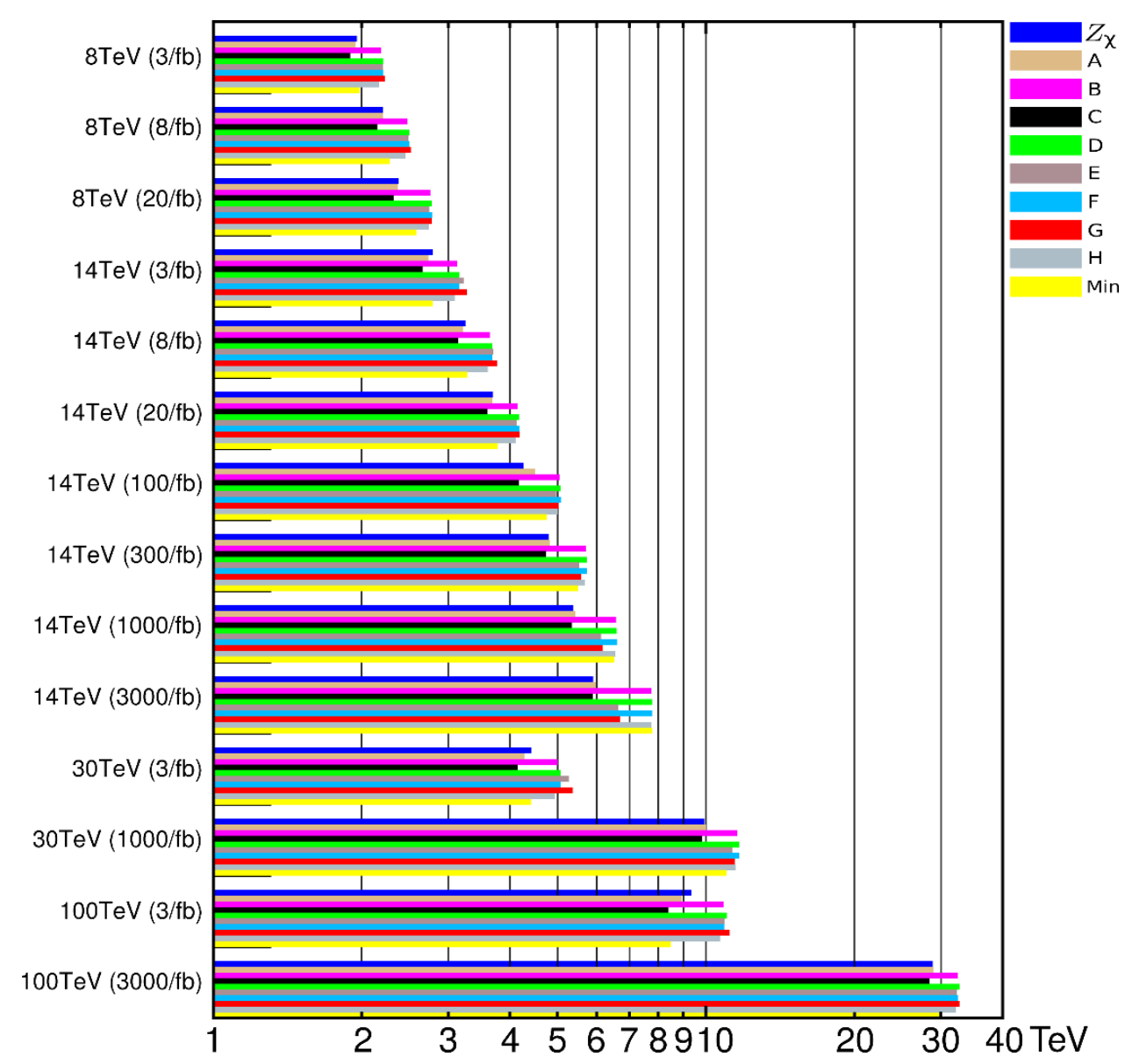

Figure 1. Projected 95\% CL exclusion limits on $M_{Z^{\prime}}$ for several 3-3-1 models by using our statistical methods. We obtain this limits by assuming that the number of observed events $n_{i}$ is equal to the SM expectation $\mu_{i}$ in every bin. We have assumed for the product acceptance $\times$ efficiency the ATLAS result for the dimuon channel as is shown in figure 1 in ref. [35].

the dimuon channel as is shown in figure 1 in [35]. The limits in figure 2 are comparable with the limits published in [36].

\section{Conclusions}

In the present work we have reported the vector and axial charges for all 3-3-1 models without exotic electric charges for leptons, known in the literature. To the best of our knowledge most of this charges were not reported before and represent a original contribution to the field. By using ATLAS data from the Drell-Yang process $p p \rightarrow Z, \gamma \rightarrow l^{+} l^{-}$ we set $95 \%$ CL lower limits for the $Z^{\prime}$ mass in every one of this models. We calculated this limits for the dimuon and the dielectron channels. Our results are in accordance with the ATLAS reported lower mass limits for the SSM, $Z_{\mathrm{SSM}}$ in every channel. By neglecting systematic uncertainties we were able to combine the two channels finding good agreement 


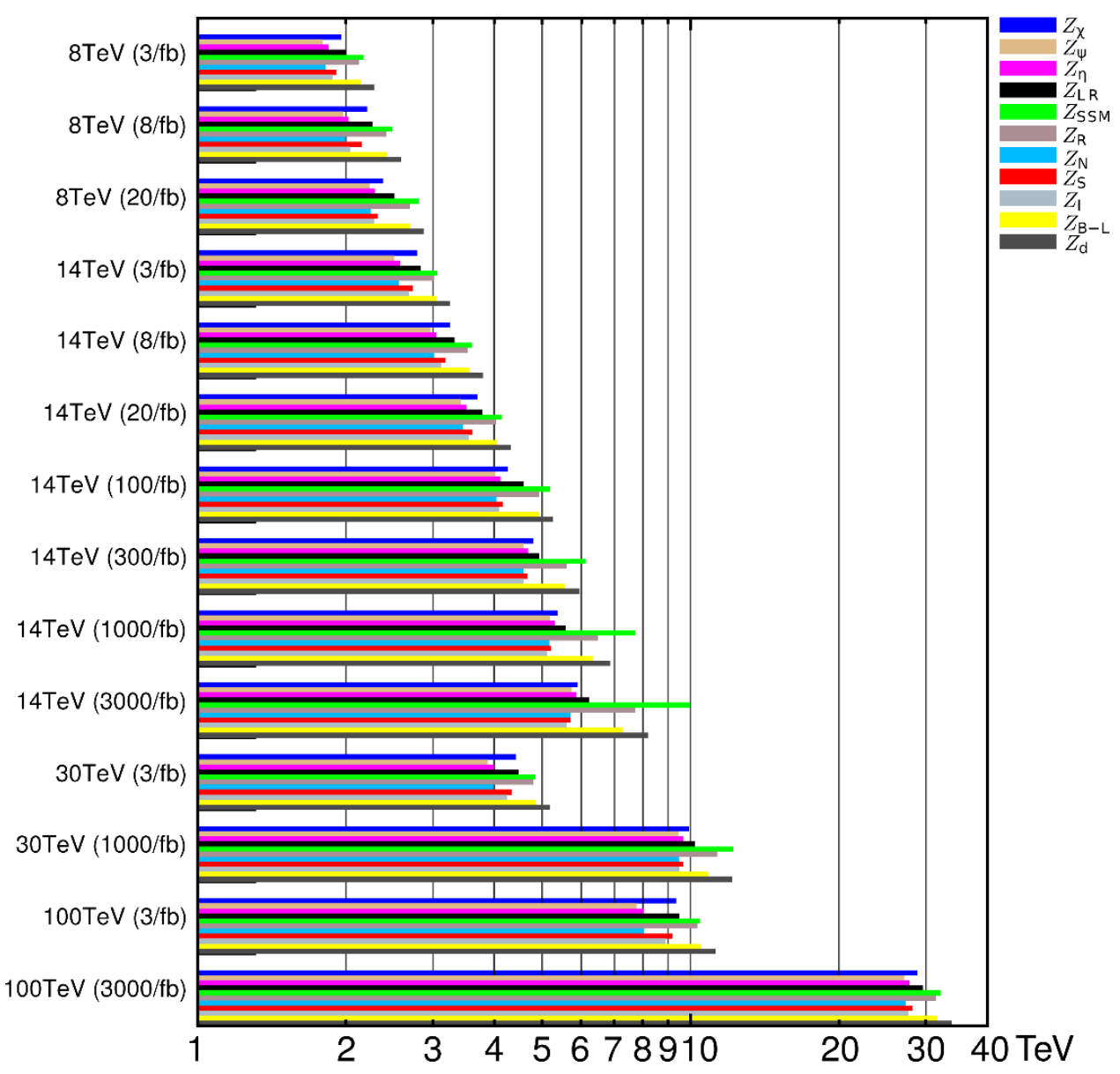

Figure 2. Projected $95 \% \mathrm{CL}$ exclusion limits on $M_{Z^{\prime}}$ for several $E_{6}$-motivated $Z^{\prime}$ models and the SSM by using our statistical methods. We obtain this limits by assuming that the number of observed events $n_{i}$ is equal to the SM expectation $\mu_{i}$ in every bin. We have assumed for the product acceptancexefficiency the ATLAS result for the dimuon channel as is shown in figure 1 in ref. [35].

with the ATLAS published results. As far as we know this is the first time that 3-3-1 models have been constrained with LHC data from ATLAS. In addition we also calculated $95 \%$ projected exclusion limits for the forthcoming LHC and VLHC energies and luminosities. As we already mentioned in the text, this projected limits are comparable with previous calculations, in particular we find that for a center of mass energy of $14 \mathrm{TeV}$ and a integrated luminosity of $100 \mathrm{fb}^{-1}$ the projected $95 \%$ CL exclusion limits for 3-3-1 models are between $4 \mathrm{TeV}$ and $5 \mathrm{TeV}$. Part of our long term goal is to present a unified phenomenological analysis for the 3-3-1 models in oder to set the relevance of the forthcoming experiments for every point in the parameter space $g_{2} \mathrm{Vs} M_{Z^{\prime}}$. We postpone to a future work a comparative study between FCNC against those coming of direct searches at hadron colliders. 


\section{Acknowledgments}

We thank financial support from "Patrimonio Autónomo Fondo Nacional de Financiamiento para la Ciencia, la Tecnología y la Innovación, Francisco José de Caldas" and "Sostenibilidad-UDEA 2014-2015". R. H. B. thanks to "Centro de Investigaciones del ITM". E.R. acknowledges financial support provided by FAPESP. We also like to thank P. Langacker for his valuable suggestions.

\section{A Differential cross-section}

The NLO differential cross-section for the DY process with a neutral gauge boson $G$ as the mediator, $p p \rightarrow G X \rightarrow l^{+} l^{-} X$, is given as [33, 62], ${ }^{7}$

$$
\begin{aligned}
\frac{d \sigma^{\mathrm{NLO}}}{d M_{l^{+} l^{-}}}= & \frac{2}{N_{c} s} M_{l^{+} l^{-}} \int d z d x_{1} \frac{1}{x_{1} z} \theta\left(1-\frac{1}{x_{1} z r_{z}^{2}}\right) \sum_{q} \hat{\sigma}_{q \bar{q} \rightarrow \ell^{+} \ell^{-}}\left(M_{l^{+} l^{-}}^{2}\right) \\
& \times\left[\left\{f_{q}^{A}\left(x_{1}, M_{l^{+} l^{-}}^{2}\right) f_{\bar{q}}^{B}\left(x_{2}, M_{l^{+} l^{-}}^{2}\right)+f_{\bar{q}}^{A}\left(x_{1}, M^{2}\right) f_{q}^{B}\left(x_{2}, M_{l^{+} l^{-}}^{2}\right)\right\}\right. \\
& \times\left\{\delta(1-z)+\frac{\alpha_{s}\left(M_{l^{+} l^{-}}^{2}\right)}{2 \pi} D_{q}(z)\right\} \\
& +\left\{f_{g}^{A}\left(x_{1}, M_{l^{+} l^{-}}^{2}\right)\left[f_{q}^{B}\left(x_{2}, M_{l^{+} l^{-}}^{2}\right)+f_{\bar{q}}^{B}\left(x_{2}, M_{l^{+} l^{-}}^{2}\right)\right]\right. \\
& \left.\left.+f_{g}^{B}\left(x_{2}, M^{2}\right)\left[f_{q}^{A}\left(x_{1}, M_{l^{+} l^{-}}^{2}\right)+f_{\bar{q}}^{A}\left(x_{1}, M_{l^{+} l^{-}}^{2}\right)\right]\right\} \times \frac{\alpha_{s}\left(M_{l^{+} l^{-}}^{2}\right)}{2 \pi} D_{g}(z)\right]
\end{aligned}
$$

where $N_{c}=3$ is the color factor, $M_{l^{+} l^{-}}$is the invariant mass of the observed lepton pair and $\sqrt{s}$ is the energy of the $p \bar{p}$ collision in the CM frame, $r_{z} \equiv \sqrt{s} / M_{l^{+} l^{-}}$, and $x_{2}^{-1} \equiv x_{1} z r_{z}^{2} \cdot f_{q / g}^{A}$ are the PDFs of the quarks and gluons coming from hadron $A . \alpha_{s}$ is the strong coupling constant, and

$$
\begin{aligned}
& D_{q}(z)=C_{F}\left[4\left(1+z^{2}\right)\left\{\frac{\log (1-z)}{1-z}\right\}_{+}-2 \frac{1+z^{2}}{1-z} \log z+\delta(1-z)\left\{\frac{2 \pi^{2}}{3}-8\right\}\right], \\
& D_{g}(z)=T_{R}\left[\left\{z^{2}+(1-z)^{2}\right\} \log \frac{(1-z)^{2}}{z}+\frac{1}{2}+3 z-\frac{7}{2} z^{2}\right],
\end{aligned}
$$

with $C_{F}=4 / 3$ and $T_{R}=1 / 2$, and the ' + ' distribution defined as

$$
\int_{0}^{1} d z g(z)\left\{\frac{\log (1-z)}{1-z}\right\}_{+} \equiv \int_{0}^{1} d z\{g(z)-g(1)\}\left\{\frac{\log (1-z)}{1-z}\right\} .
$$

At parton level, the expression for the hard scattering cross-section of the process $q \bar{q} \rightarrow$ $\ell^{+} \ell^{-}$, is

$$
\begin{aligned}
& \hat{\sigma}_{q \bar{q} \rightarrow \ell^{+} \ell^{-}}\left(M^{2}\right)=\int_{-1}^{1} \frac{d \hat{\sigma}}{d \cos \theta^{*}} d \cos \theta^{*} \\
& \quad=\int_{-1}^{1} \frac{d \cos \theta^{*}}{128 \pi M^{2}}\left\{\left(\left|A_{L L}\right|^{2}+\left|A_{R R}\right|^{2}\right)\left(1+\cos \theta^{*}\right)^{2}+\left(\left|A_{L R}\right|^{2}+\left|A_{R L}\right|^{2}\right)\left(1-\cos \theta^{*}\right)^{2}\right\},
\end{aligned}
$$

\footnotetext{
${ }^{7}$ The integration over $z$ is carried out as $\int_{0}^{(1+\epsilon)} d z \delta(1-z)=1$.
} 
where $\theta^{*}$ is the polar angle in the CM frame, and

$$
A_{i j}=-Q(q) e^{2}+\frac{g_{1}^{2} \epsilon_{1 i}(q) \epsilon_{1 j}(\ell) M^{2}}{M^{2}-M_{Z}^{2}+i M_{Z} \Gamma_{Z}}+\frac{g_{2}^{2} \epsilon_{2 i}(q) \epsilon_{2 j}(\ell) M^{2}}{M^{2}-M_{Z^{\prime}}^{2}+i M_{Z^{\prime}} \Gamma_{Z^{\prime}}},
$$

where $i, j$ run over $L, R . Q(q)$ is the electric charge of the quark and $e=g \sin \theta_{W} \cdot M_{Z, Z^{\prime}}$ and $\Gamma_{Z, Z^{\prime}}$ are the masses and total decay widths of the $Z$ and $Z^{\prime}$ bosons.

$$
\epsilon_{1 L}(f)=T_{3}(f)-Q(f) \sin ^{2} \theta_{W}, \quad \epsilon_{1 R}(f)=-Q(f) \sin ^{2} \theta_{W},
$$

are the effective couplings of the ordinary $Z$ to fermion $f$ entering with coupling strength, $g_{1}=g / \cos \theta_{W}=0.7433$. As for the $Z^{\prime}$ coupling strength, for $E_{6}$ we employ the (one-loop) unification value [8], $g_{2}=\sqrt{5 / 3} \sin \theta_{W} g_{1}=0.4615$; for 3-3-1 models see appendix B. The decay width, $\Gamma_{Z^{\prime}}$, given in eq. (A.5), is the sum of the partial decay widths of the $Z^{\prime}$ boson into all the fermions it couples to. The partial decay width into a Dirac fermion pair is written as [63]

$\Gamma_{Z^{\prime} \rightarrow f \bar{f}}\left(M_{l^{+} l^{-}}^{2}\right)=\frac{g_{2}^{2} M_{Z^{\prime}}}{24 \pi} \sqrt{1-\frac{4 M_{f}^{2}}{M_{Z^{\prime}}^{2}}}\left[\left(1-\frac{M_{f}^{2}}{M_{Z^{\prime}}^{2}}\right)\left(\epsilon_{2 L}^{2}(f)+\epsilon_{2 R}^{2}(f)\right)-\frac{6 M_{f}^{2}}{M_{l^{+} l^{-}}^{2}} \epsilon_{2 L}(f) \epsilon_{2 R}(f)\right] \frac{M_{l^{+} l^{-}}^{2}}{M_{Z^{\prime}}^{2}}$,

where $M_{f}$ is the mass of the final-state fermion. We add the factor $M^{2} / M_{Z^{\prime}}^{2}$ to get an ' $\hat{s}$ '-dependent $Z^{\prime}$-width [64]. For the range of $M_{Z^{\prime}}$ of interest here, $M_{f} \ll M_{Z^{\prime}}$ for SM fermions, and the above expression becomes independent of the fermion masses.

\section{B The 3-3-1 couplings}

For the SM extended by a U(1)' extra factor, the neutral current interactions of the fermions are described by the Hamiltonian

$$
H_{N C}=\sum_{i=1}^{2} g_{i} Z_{i \mu}^{0} \sum_{f} \bar{f} \gamma^{\mu}\left(\epsilon_{i L}(f) P_{L}+\epsilon_{i R}(f) P_{R}\right) f,
$$

where $f$ runs over all the SM fermions in the low energy Neutral Current (NC) effective Hamiltonian $H_{N C}$, and $P_{L}=\left(1-\gamma_{5}\right) / 2$ and $P_{R}=\left(1+\gamma_{5}\right) / 2$. For 3-3-1 models, the ralationship between $g_{1}$ and $g_{2}$ is model dependent, but for all the cases we can write

$$
H_{N C}=\frac{g}{2 \cos \theta_{W}} \sum_{i=1}^{2} Z_{i \mu}^{0} \sum_{f} \bar{f} \gamma^{\mu}\left(g_{i V}(f)-g_{i A}(f) \gamma_{5}\right) f
$$

where the chiral couplings $\epsilon_{i L}(f)$ and $\epsilon_{i R}(f)$ are linear combinations of the vector $g_{i V}(f)$ and axial $g_{i A}(f)$ charges given by $\epsilon_{i L}(f)=\left[g_{i V}(f)+g_{i A}(f)\right] / 2$ and $\epsilon_{i R}(f)=\left[g_{i V}(f)-g_{i A}(f)\right] / 2$.

The physical fields in the former expressions are:

$$
\begin{aligned}
& Z_{1}^{\mu}=Z^{\mu} \cos \theta+Z^{\prime \mu} \sin \theta, \\
& Z_{2}^{\mu}=-Z^{\mu} \sin \theta+Z^{\prime \mu} \cos \theta,
\end{aligned}
$$




\begin{tabular}{|ccc|}
\hline Field & $g_{2 V}(f)$ & $g_{2 A}(f)$ \\
\hline$\nu_{\alpha}$ & $\left(\frac{1}{2}-\sin ^{2} \theta_{W}\right) \frac{1}{\delta}$ & $\left(\frac{1}{2}-\sin ^{2} \theta_{W}\right) \frac{1}{\delta}$ \\
$e_{\alpha}$ & $-\left(-\frac{1}{2}+2 \sin ^{2} \theta_{W}\right) \frac{1}{\delta}$ & $\frac{1}{2} \frac{1}{\delta}$ \\
$u_{i}$ & $\left(-\frac{1}{2}+\frac{4}{3} \sin ^{2} \theta_{W}\right) \frac{1}{\delta}$ & $-\frac{1}{2} \frac{1}{\delta}$ \\
$u_{3}$ & $\left(\frac{1}{2}+\frac{1}{3} \sin ^{2} \theta_{W}\right) \frac{1}{\delta}$ & $-\left(-\frac{1}{2}+\sin ^{2} \theta_{W}\right) \frac{1}{\delta}$ \\
$d_{i}$ & $-\left(\frac{1}{2}-\frac{1}{3} \sin ^{2} \theta_{W}\right) \frac{1}{\delta}$ & $-\left(\frac{1}{2}-\sin ^{2} \theta_{W}\right) \frac{1}{\delta}$ \\
$d_{3}$ & $-\left(-\frac{1}{2}+\frac{2}{3} \sin ^{2} \theta_{W}\right) \frac{1}{\delta}$ & $\frac{1}{2} \frac{1}{\delta}$ \\
\hline
\end{tabular}

Table 4. Model A, $\alpha=1,2,3$, and $i=1,2$.

\begin{tabular}{|ccc|}
\hline Field & $g_{2 V}(f)$ & $g_{2 A}(f)$ \\
\hline$\nu_{\alpha}$ & $-\frac{1}{2} \frac{1}{\delta}$ & $-\frac{1}{2} \frac{1}{\delta}$ \\
$e_{\alpha}$ & $-\left(\frac{1}{2}+\sin ^{2} \theta_{W}\right) \frac{1}{\delta}$ & $-\left(\frac{1}{2}-\sin ^{2} \theta_{W}\right) \frac{1}{\delta}$ \\
$u_{i}$ & $\left(\frac{1}{2}+\frac{1}{3} \sin ^{2} \theta_{W}\right) \frac{1}{\delta}$ & $-\left(-\frac{1}{2}+\sin ^{2} \theta_{W}\right) \frac{1}{\delta}$ \\
$u_{3}$ & $\left(-\frac{1}{2}+\frac{4}{3} \sin ^{2} \theta_{W}\right) \frac{1}{\delta}$ & $-\frac{1}{2} \frac{1}{\delta}$ \\
$d_{i}$ & $\left(\frac{1}{2}-\frac{2}{3} \sin ^{2} \theta_{W}\right) \frac{1}{\delta}$ & $\frac{1}{2} \frac{1}{\delta}$ \\
$d_{3}$ & $\left(-\frac{1}{2}+\frac{1}{3} \sin ^{2} \theta_{W}\right) \frac{1}{\delta}$ & $-\left(\frac{1}{2}-\sin ^{2} \theta_{W}\right) \frac{1}{\delta}$ \\
\hline
\end{tabular}

Table 5. Model B, $\alpha=1,2,3$, and $i=1,2$.

\begin{tabular}{|ccc|}
\hline Field & $g_{2 V}(f)$ & $g_{2 A}(f)$ \\
\hline$\nu_{3}$ & $-\frac{1}{2} \frac{1}{\delta}$ & $-\frac{1}{2} \frac{1}{\delta}$ \\
$\nu_{i}$ & $-\left(-\frac{1}{2}+\sin ^{2} \theta_{W}\right) \frac{1}{\delta}$ & $-\left(-\frac{1}{2}+\sin ^{2} \theta_{W}\right) \frac{1}{\delta}$ \\
$e_{1}$ & $-\left(\frac{1}{2}+\sin ^{2} \theta_{W}\right) \frac{1}{\delta}$ & $-\left(\frac{1}{2}-\sin ^{2} \theta_{W}\right) \frac{1}{\delta}$ \\
$e_{2}$ & $-\left(-\frac{1}{2}+2 \sin ^{2} \theta_{W}\right) \frac{1}{\delta}$ & $\frac{1}{2} \frac{1}{\delta}$ \\
$e_{3}$ & $-3\left(-\frac{1}{2}+\sin ^{2} \theta_{W}\right) \frac{1}{\delta}$ & $\left(\frac{1}{2}-\cos ^{2} \theta_{w}\right) \frac{1}{\delta}$ \\
$u_{i}$ & $-\left(\frac{1}{2}-\frac{4}{3} \sin ^{2} \theta_{W}\right) \frac{1}{\delta}$ & $-\frac{1}{2} \frac{1}{\delta}$ \\
$u_{3}$ & $-\left(-\frac{1}{2}-\frac{1}{3} \sin ^{2} \theta_{W}\right) \frac{1}{\delta}$ & $-\left(-\frac{1}{2}+\sin ^{2} \theta_{W}\right) \frac{1}{\delta}$ \\
$d_{i}$ & $-\left(\frac{1}{2}-\frac{1}{3} \sin ^{2} \theta_{W}\right) \frac{1}{\delta}$ & $-\left(\frac{1}{2}-\sin ^{2} \theta_{W}\right) \frac{1}{\delta}$ \\
$d_{3}$ & $-\left(-\frac{1}{2}+\frac{2}{3} \sin ^{2} \theta_{W}\right) \frac{1}{\delta}$ & $\frac{1}{2} \frac{1}{\delta}$ \\
\hline
\end{tabular}

Table 6. Model $\mathrm{C}$ and $i=1,2$.

where $Z^{\mu}$ and $Z^{\prime \mu}$ are the weak basis states such that $Z^{\mu}$ is identified with the neutral gauge boson of the SM. At a first approximation we have taken $\theta=0$.

For the numerical calculations we use the expressions in tables 4 to 12, where most of the values in the tables are being presented for the first time in the literature. We have also used: $M_{W}=80.401 \mathrm{GeV}, M_{Z}=91.188 \mathrm{GeV}, \cos \theta_{W}=M_{W} / M_{Z}, \delta=\sqrt{4 \cos ^{2} \theta_{W}-1}$ and $g_{1} \equiv g / \cos \theta_{W}=0.7433$. 


\begin{tabular}{|ccc|}
\hline Field & $g_{2 V}(f)$ & $g_{2 A}(f)$ \\
\hline$\nu_{i}$ & $-\frac{1}{2} \frac{1}{\delta}$ & $-\frac{1}{2} \frac{1}{\delta}$ \\
$\nu_{3}$ & $\left(\frac{1}{2}-\sin ^{2} \theta_{W}\right) \frac{1}{\delta}$ & $\left(\frac{1}{2}-\sin ^{2} \theta_{W}\right) \frac{1}{\delta}$ \\
$e_{i}$ & $-\left(\frac{1}{2}+\sin ^{2} \theta_{W}\right) \frac{1}{\delta}$ & $-\left(\frac{1}{2}-\sin ^{2} \theta_{W}\right) \frac{1}{\delta}$ \\
$e_{3}$ & $-\left(-\frac{1}{2}+2 \sin ^{2} \theta_{W}\right) \frac{1}{\delta}$ & $\frac{1}{2} \frac{1}{\delta}$ \\
$u_{i}$ & $\left(\frac{1}{2}+\frac{1}{3} \sin ^{2} \theta_{W}\right) \frac{1}{\delta}$ & $-\left(-\frac{1}{2}+\sin ^{2} \theta_{W}\right) \frac{1}{\delta}$ \\
$u_{3}$ & $-\left(\frac{1}{2}-\frac{4}{3} \sin ^{2} \theta_{W}\right) \frac{1}{\delta}$ & $-\frac{1}{2} \frac{1}{\delta}$ \\
$d_{i}$ & $-\left(-\frac{1}{2}+\frac{2}{3} \sin ^{2} \theta_{W}\right) \frac{1}{\delta}$ & $\frac{1}{2} \frac{1}{\delta}$ \\
$d_{3}$ & $-\left(\frac{1}{2}-\frac{1}{3} \sin ^{2} \theta_{W}\right) \frac{1}{\delta}$ & $-\left(\frac{1}{2}-\sin ^{2} \theta_{W}\right) \frac{1}{\delta}$ \\
\hline
\end{tabular}

Table 7. Model D and $i=1,2$.

\begin{tabular}{|ccc|}
\hline Field & $g_{2 V}(f)$ & $g_{2 A}(f)$ \\
\hline$\nu_{i}$ & $\left(\frac{1}{2}-\sin ^{2} \theta_{W}\right) \frac{1}{\delta}$ & $\left(\frac{1}{2}-\sin ^{2} \theta_{W}\right) \frac{1}{\delta}$ \\
$\nu_{3}$ & $-\frac{1}{2} \frac{1}{\delta}$ & $-\frac{1}{2} \frac{1}{\delta}$ \\
$e_{i}$ & $\left(\frac{3}{2}-3 \sin ^{2} \theta_{W}\right) \frac{1}{\delta}$ & $\left(\frac{1}{2}-\cos ^{2} \theta_{W}\right) \frac{1}{\delta}$ \\
$e_{3}$ & $-\left(\frac{1}{2}+\sin ^{2} \theta_{W}\right) \frac{1}{\delta}$ & $\left(-\frac{1}{2}+\sin ^{2} \theta_{W}\right) \frac{1}{\delta}$ \\
$u_{i}$ & $\left(-\frac{1}{2}+\frac{4}{3} \sin ^{2} \theta_{W}\right) \frac{1}{\delta}$ & $-\frac{1}{2} \frac{1}{\delta}$ \\
$u_{3}$ & $\left(\frac{1}{2}+\frac{1}{3} \sin ^{2} \theta_{W}\right) \frac{1}{\delta}$ & $\left(\frac{1}{2}-\sin ^{2} \theta_{W}\right) \frac{1}{\delta}$ \\
$d_{i}$ & $\left(-\frac{1}{2}+\frac{1}{3} \sin ^{2} \theta_{W}\right) \frac{1}{\delta}$ & $\left(-\frac{1}{2}+\sin ^{2} \theta_{W}\right) \frac{1}{\delta}$ \\
$d_{3}$ & $\left(\frac{1}{2}-\frac{2}{3} \sin ^{2} \theta_{W}\right) \frac{1}{\delta}$ & $\frac{1}{2} \frac{1}{\delta}$ \\
\hline
\end{tabular}

Table 8. Model E and $i=1,2$.

\begin{tabular}{|ccc|}
\hline Field & $g_{2 V}(f)$ & $g_{2 A}(f)$ \\
\hline$\nu_{i}$ & $-\frac{1}{2} \frac{1}{\delta}$ & $-\frac{1}{2} \frac{1}{\delta}$ \\
$\nu_{3}$ & $\left(\frac{1}{2}-\sin ^{2} \theta_{W}\right) \frac{1}{\delta}$ & $\left(\frac{1}{2}-\sin ^{2} \theta_{W}\right) \frac{1}{\delta}$ \\
$e_{i}$ & $\left(-\frac{1}{2}-\sin ^{2} \theta_{W}\right) \frac{1}{\delta}$ & $\left(-\frac{1}{2}+\sin ^{2} \theta_{W}\right) \frac{1}{\delta}$ \\
$e_{3}$ & $\left(\frac{3}{2}-3 \sin ^{2} \theta_{W}\right) \frac{1}{\delta}$ & $\left(\frac{1}{2}-\cos ^{2} \theta_{W}\right) \frac{1}{\delta}$ \\
$u_{i}$ & $\left(\frac{1}{2}+\frac{1}{3} \sin ^{2} \theta_{W}\right) \frac{1}{\delta}$ & $\left(\frac{1}{2}-\sin ^{2} \theta_{W}\right) \frac{1}{\delta}$ \\
$u_{3}$ & $\left(-\frac{1}{2}+\frac{4}{3} \sin ^{2} \theta_{W}\right) \frac{1}{\delta}$ & $-\frac{1}{2} \frac{1}{\delta}$ \\
$d_{i}$ & $\left(\frac{1}{2}-\frac{2}{3} \sin ^{2} \theta_{W}\right) \frac{1}{\delta}$ & $\frac{1}{2} \frac{1}{\delta}$ \\
$d_{3}$ & $\left(-\frac{1}{2}+\frac{1}{3} \sin ^{2} \theta_{W}\right) \frac{1}{\delta}$ & $\left(-\frac{1}{2}+\sin ^{2} \theta_{W}\right) \frac{1}{\delta}$ \\
\hline
\end{tabular}

Table 9. Model $\mathrm{F}$ and $i=1,2$. 


\begin{tabular}{|ccc|}
\hline Field & $g_{2 V}(f)$ & $g_{2 A}(f)$ \\
\hline$\nu_{\alpha}$ & $\left(\frac{1}{2}-\sin ^{2} \theta_{W}\right) \frac{1}{\delta}$ & $\left(\frac{1}{2}-\sin ^{2} \theta_{W}\right) \frac{1}{\delta}$ \\
$e_{\alpha}$ & $3\left(\frac{1}{2}-\sin ^{2} \theta_{W}\right) \frac{1}{\delta}$ & $\left(\frac{1}{2}-\cos ^{2} \theta_{W}\right) \frac{1}{\delta}$ \\
$u_{\alpha}$ & $-\left(\frac{1}{2}-\frac{4}{3} \sin ^{2} \theta_{W}\right) \frac{1}{\delta}$ & $-\frac{1}{2} \frac{1}{\delta}$ \\
$d_{\alpha}$ & $-\left(\frac{1}{2}-\frac{1}{3} \sin ^{2} \theta_{W}\right) \frac{1}{\delta}$ & $-\frac{1}{2} \cos 2 \theta_{W} \frac{1}{\delta}$ \\
\hline
\end{tabular}

Table 10. Model $\mathrm{G}$ and $\alpha=1,2,3$,

\begin{tabular}{|ccc|}
\hline Field & $g_{2 V}(f)$ & $g_{2 A}(f)$ \\
\hline$\nu_{\alpha}$ & $-\frac{1}{2} \frac{1}{\delta}$ & $-\frac{1}{2} \frac{1}{\delta}$ \\
$e_{\alpha}$ & $-\left(\frac{1}{2}+\sin ^{2} \theta_{W}\right) \frac{1}{\delta}$ & $\left(-\frac{1}{2}+\sin ^{2} \theta_{W}\right) \frac{1}{\delta}$ \\
$u_{\alpha}$ & $\left(\frac{1}{2}+\frac{1}{3} \sin ^{2} \theta_{W}\right) \frac{1}{\delta}$ & $\left(\frac{1}{2}-\sin ^{2} \theta_{W}\right) \frac{1}{\delta}$ \\
$d_{\alpha}$ & $\left(\frac{1}{2}-\frac{2}{3} \sin ^{2} \theta_{W}\right) \frac{1}{\delta}$ & $\frac{1}{2} \frac{1}{\delta}$ \\
\hline
\end{tabular}

Table 11. Model $\mathrm{H}$ and $\alpha=1,2,3$,

\begin{tabular}{|ccc|}
\hline Field & $g_{2 V}(f)$ & $g_{2 A}(f)$ \\
\hline$\nu_{\alpha}$ & $-\frac{\sqrt{1-4 \sin ^{2} \theta_{W}}}{2 \sqrt{3}}$ & $-\frac{\sqrt{1-4 \sin ^{2} \theta_{W}}}{2 \sqrt{3}}$ \\
$e_{\alpha}$ & $-\frac{\sqrt{3\left(1-4 \sin ^{2} \theta_{W}\right)}}{2}$ & $+\frac{\sqrt{1-4 \sin ^{2} \theta_{W}}}{2 \sqrt{3}}$ \\
$u_{i}$ & $-\frac{-1+6 \sin ^{2} \theta_{W}}{2 \sqrt{3\left(1-4 \sin ^{2} \theta_{W}\right)}}$ & $+\frac{1+2 \sin ^{2} \theta_{W}}{2 \sqrt{3\left(1-4 \sin ^{2} \theta_{W}\right)}}$ \\
$t$ & $-\frac{1+4 \sin ^{2} \theta_{W}}{2 \sqrt{3\left(1-4 \sin ^{2} \theta_{W}\right)}}$ & $-\frac{1-4 \sin ^{2} \theta_{W}}{\sqrt{3\left(1-4 \sin ^{2} \theta_{W}\right)}}$ \\
$d_{i}$ & $+\frac{1}{2 \sqrt{3\left(1-4 \sin ^{2} \theta_{W}\right)}}$ & $-\frac{-1+4 \sin ^{2} \theta_{W}}{2 \sqrt{3\left(1-4 \sin ^{2} \theta_{W}\right)}}$ \\
$b$ & $-\frac{1-2 \sin ^{2} \theta_{W}}{2 \sqrt{3\left(1-4 \sin ^{2} \theta_{W}\right)}}$ & $-\frac{1+2 \sin ^{2} \theta_{W}}{2 \sqrt{3\left(1-4 \sin ^{2} \theta_{W}\right)}}$ \\
\hline
\end{tabular}

Table 12. Minimal Model: Pleitez-Frampton [65]. $\alpha=1,2,3$, and $i=1,2$. 
Open Access. This article is distributed under the terms of the Creative Commons Attribution License (CC-BY 4.0), which permits any use, distribution and reproduction in any medium, provided the original author(s) and source are credited.

\section{References}

[1] J. Donoghue, E. Golowich and B.R. Holstein, Dynamics of the standard model, Cambridge University Press, Cambridge U.K. (1992).

[2] P. Langacker and M. Plümacher, Flavor changing effects in theories with a heavy $Z^{\prime}$ boson with family nonuniversal couplings, Phys. Rev. D 62 (2000) 013006 [hep-ph/0001204] [INSPIRE].

[3] R. Slansky, Group Theory for Unified Model Building, Phys. Rept. 79 (1981) 1 [inSPIRE].

[4] P. Langacker, The Physics of Heavy Z' Gauge Bosons, Rev. Mod. Phys. 81 (2009) 1199 [arXiv:0801.1345] [INSPIRE].

[5] T. Appelquist, B.A. Dobrescu and A.R. Hopper, Nonexotic neutral gauge bosons, Phys. Rev. D 68 (2003) 035012 [hep-ph/0212073] [INSPIRE].

[6] M. Carena, A. Daleo, B.A. Dobrescu and T.M.P. Tait, $Z^{\prime}$ gauge bosons at the Tevatron, Phys. Rev. D 70 (2004) 093009 [hep-ph/0408098] [InSPIRE].

[7] F. Gursey, P. Ramond and P. Sikivie, A Universal Gauge Theory Model Based on E6, Phys. Lett. B 60 (1976) 177 [inSPIRE].

[8] R.W. Robinett and J.L. Rosner, Mass Scales in Grand Unified Theories, Phys. Rev. D 26 (1982) 2396 [INSPIRE].

[9] J. Erler, P. Langacker, S. Munir and E. Rojas, Z' Bosons at Colliders: a Bayesian Viewpoint, JHEP 11 (2011) 076 [arXiv:1103.2659] [INSPIRE].

[10] M. Singer, J.W.F. Valle and J. Schechter, Canonical Neutral Current Predictions From the Weak Electromagnetic Gauge Group $\mathrm{SU}(3) \times \mathrm{U}(1)$, Phys. Rev. D 22 (1980) 738 [inSPIRE].

[11] F. Pisano and V. Pleitez, An $\mathrm{SU}(3) \times \mathrm{U}(1)$ model for electroweak interactions, Phys. Rev. D 46 (1992) 410 [hep-ph/9206242] [INSPIRE].

[12] P. Frampton, Chiral dilepton model and the flavor question, Phys. Rev. Lett. 69 (1992) 2889.

[13] J.C. Montero, F. Pisano and V. Pleitez, Neutral currents and GIM mechanism in $\mathrm{SU}(3)_{L} \times \mathrm{U}(1)_{N}$ models for electroweak interactions, Phys. Rev. D 47 (1993) 2918 [hep-ph/9212271] [INSPIRE].

[14] R. Foot, O.F. Hernandez, F. Pisano and V. Pleitez, Lepton masses in an $\mathrm{SU}(3)_{L} \times \mathrm{U}(1)_{N}$ gauge model, Phys. Rev. D 47 (1993) 4158 [hep-ph/9207264] [INSPIRE].

[15] R. Foot, H.N. Long and T.A. Tran, $\mathrm{SU}(3)_{L} \otimes \mathrm{U}(1)_{N}$ and $\mathrm{SU}(4)_{L} \otimes \mathrm{U}(1)_{N}$ gauge models with right-handed neutrinos, Phys. Rev. D 50 (1994) R34.

[16] M. Ozer, $\mathrm{SU}(3)_{L} \times \mathrm{U}(1)_{X}$ model of the electroweak interactions without exotic quarks, Phys. Rev. D 54 (1996) 1143 [INSPIRE].

[17] W.A. Ponce, J.B. Florez and L.A. Sanchez, Analysis of $\mathrm{SU}(3)_{c} \times \mathrm{SU}(3)_{L} \times \mathrm{U}(1)_{X}$ local gauge theory, Int. J. Mod. Phys. A 17 (2002) 643 [hep-ph/0103100] [InSPIRE].

[18] W.A. Ponce, Y. Giraldo and L.A. Sanchez, Minimal scalar sector of 3-3-1 models without exotic electric charges, Phys. Rev. D 67 (2003) 075001 [hep-ph/0210026] [INSPIRE]. 
[19] M. Ozer, GIM mechanism and its consequences in the $\mathrm{SU}(3)_{L} \times \mathrm{U}(1)_{X}$ models of electroweak interactions, Phys. Rev. D 54 (1996) 4561.

[20] J.C. Montero, C.C. Nishi, V. Pleitez, O. Ravinez and M.C. Rodriguez, Soft CP-violation in $K$ meson systems, Phys. Rev. D 73 (2006) 016003 [hep-ph/0511100] [INSPIRE].

[21] A.J. Buras, F. Schwab and S. Uhlig, Waiting for precise measurements of $K^{+} \rightarrow \pi^{+} \nu \bar{\nu}$ and $K_{-} L \rightarrow \pi^{0} \nu \bar{\nu}$, Rev. Mod. Phys. 80 (2008) 965 [hep-ph/0405132] [INSPIRE].

[22] A.J. Buras, F. De Fazio, J. Girrbach and M.V. Carlucci, The Anatomy of Quark Flavour Observables in 331 Models in the Flavour Precision Era, JHEP 02 (2013) 023 [arXiv: 1211.1237] [INSPIRE].

[23] A.J. Buras and J. Girrbach, Towards the Identification of New Physics through Quark Flavour Violating Processes, Rept. Prog. Phys. 77 (2014) 086201 [arXiv:1306.3775] [INSPIRE].

[24] A.J. Buras, F. De Fazio and J. Girrbach-Noe, Z-Z' mixing and Z-mediated FCNCs in $\mathrm{SU}(3)_{C} \times \mathrm{SU}(3)_{L} \times \mathrm{U}(1)_{X}$ models, JHEP 08 (2014) 039 [arXiv: 1405.3850] [inSPIRE].

[25] C. Promberger, S. Schatt and F. Schwab, Flavor Changing Neutral Current Effects and CP-violation in the Minimal 3-3-1 Model, Phys. Rev. D 75 (2007) 115007 [hep-ph/0702169] [INSPIRE].

[26] J.M. Cabarcas, J. Duarte and J.-A. Rodriguez, Sources of FCNC in $\mathrm{SU}(3)_{C} \otimes \mathrm{SU}(3)_{L} \otimes \mathrm{U}(1)_{X}$ Models, Adv. High Energy Phys. 2012 (2012) 657582 [arXiv:1111.0315] [INSPIRE].

[27] A.C.B. Machado, J.C. Montero and V. Pleitez, Flavor-changing neutral currents in the minimal 3-3-1 model revisited, Phys. Rev. D 88 (2013) 113002 [arXiv:1305.1921] [INSPIRE].

[28] R. Martinez and F. Ochoa, Constraints on 3-3-1 models with electroweak $Z$ pole observables and Z' search at the LHC, Phys. Rev. D 90 (2014) 015028 [arXiv:1405.4566] [INSPIRE].

[29] C.A. de Sousa Pires and O.P. Ravinez, Charge quantization in a chiral bilepton gauge model, Phys. Rev. D 58 (1998) 035008 [hep-ph/9803409] [INSPIRE].

[30] P.V. Dong and H.N. Long, Electric charge quantization in $\mathrm{SU}(3)_{C} \times \mathrm{SU}(3)_{L} \times \mathrm{U}(1)_{X}$ models, Int. J. Mod. Phys. A 21 (2006) 6677 [hep-ph/0507155] [inSPIRE].

[31] P.B. Pal, The Strong CP question in $\mathrm{SU}(3)_{c} \times \mathrm{SU}(3)_{L} \times \mathrm{U}(1)_{N}$ models, Phys. Rev. D 52 (1995) 1659 [hep-ph/9411406] [INSPIRE].

[32] CDF collaboration, T. Aaltonen et al., First Run II Measurement of the W Boson Mass, Phys. Rev. D 77 (2008) 112001 [arXiv: 0708.3642] [INSPIRE].

[33] J. Erler, P. Langacker, S. Munir and E. Rojas, Z' Bosons from E(6): Collider and Electroweak Constraints, arXiv:1108.0685.

[34] H.-L. Lai et al., New parton distributions for collider physics, Phys. Rev. D 82 (2010) 074024 [arXiv: 1007.2241] [INSPIRE].

[35] ATLAS collaboration, Search for high-mass dilepton resonances in pp collisions at $\sqrt{s}=8 \mathrm{TeV}$ with the ATLAS detector, Phys. Rev. D 90 (2014) 052005 [arXiv:1405.4123] [INSPIRE].

[36] S. Godfrey and T. Martin, Z' Discovery Reach at Future Hadron Colliders: A Snowmass White Paper, arXiv:1309.1688. 
[37] T.G. Rizzo, Exploring new gauge bosons at a 100 TeV collider, Phys. Rev. D 89 (2014) 095022 [arXiv: 1403.5465] [INSPIRE].

[38] R.A. Diaz, R. Martinez and F. Ochoa, $\mathrm{SU}(3)_{c} \times \mathrm{SU}(3)_{L} \times \mathrm{U}(1)_{X}$ models for beta arbitrary and families with mirror fermions, Phys. Rev. D 72 (2005) 035018 [hep-ph/0411263] [INSPIRE].

[39] A.E. Carcamo Hernandez, R. Martinez and F. Ochoa, $Z$ and $Z$ ' decays with and without FCNC in 331 models, Phys. Rev. D 73 (2006) 035007 [hep-ph/0510421] [INSPIRE].

[40] V. Pleitez and M.D. Tonasse, Neutrinoless double- $\beta$ decay in an $\mathrm{SU}(3)_{L} \times \mathrm{U}(1)_{N}$ model, Phys. Rev. D 48 (1993) 5274 [hep-ph/9302201] [INSPIRE].

[41] D. Ng, The Electroweak theory of $\mathrm{SU}(3) \times \mathrm{U}(1)$, Phys. Rev. D 49 (1994) 4805 [hep-ph/9212284] [INSPIRE].

[42] L. Epele, H. Fanchiotti, C. Garcia Canal and D. Gomez Dumm, Spontaneous CP violation in an $\mathrm{SU}(3)_{L} \otimes \mathrm{U}(1)_{Y}$ electroweak model, Phys. Lett. B 343 (1995) 291.

[43] D.A. Gutierrez, W.A. Ponce and L.A. Sanchez, Phenomenology of the $\mathrm{SU}(3)_{c} \times \mathrm{SU}(3)_{L} \times \mathrm{U}(1)_{X}$ model with right-handed neutrinos, Eur. Phys. J. C 46 (2006) 497 [hep-ph/0411077] [INSPIRE].

[44] W.A. Ponce and O. Zapata, Lepton masses and mixing without Yukawa hierarchies, Phys. Rev. D 74 (2006) 093007 [hep-ph/0611082] [INSPIRE].

[45] D.L. Anderson and M. Sher, 3-3-1 models with unique lepton generations, Phys. Rev. D 72 (2005) 095014 [hep-ph/0509200] [INSPIRE].

[46] L.A. Sanchez, W.A. Ponce and R. Martinez, $\mathrm{SU}(3)_{c} \times \mathrm{SU}(3)(L) \times \mathrm{U}(1)_{X}$ as an $E_{6}$ subgroup, Phys. Rev. D 64 (2001) 075013 [hep-ph/0103244] [InSPIRE].

[47] R. Martinez, W.A. Ponce and L.A. Sanchez, $\mathrm{SU}(3)_{c} \times \mathrm{SU}(3)_{L} \times U(1)_{X}$ as an $\mathrm{SU}(6) \times \mathrm{U}(1)_{X}$ subgroup, Phys. Rev. D 65 (2002) 055013 [hep-ph/0110246] [INSPIRE].

[48] E. Witten, Symmetry Breaking Patterns in Superstring Models, Nucl. Phys. B 258 (1985) 75 [INSPIRE].

[49] J.C. Pati and A. Salam, Lepton Number as the Fourth Color, Phys. Rev. D 10 (1974) 275 [Erratum ibid. D 11 (1975) 703].

[50] R.N. Mohapatra and J.C. Pati, Left-Right Gauge Symmetry and an Isoconjugate Model of CP-violation, Phys. Rev. D 11 (1975) 566 [INSPIRE].

[51] R.N. Mohapatra and J.C. Pati, A Natural Left-Right Symmetry, Phys. Rev. D 11 (1975) 2558 [INSPIRE].

[52] E. Ma, Neutrino masses in an extended gauge model with $E_{6}$ particle content, Phys. Lett. B 380 (1996) 286 [hep-ph/9507348] [INSPIRE].

[53] S.F. King, S. Moretti and R. Nevzorov, Theory and phenomenology of an exceptional supersymmetric standard model, Phys. Rev. D 73 (2006) 035009 [hep-ph/0510419] [INSPIRE].

[54] J. Erler, P. Langacker and T.-j. Li, The Z-Z' mass hierarchy in a supersymmetric model with a secluded U(1)-prime breaking sector, Phys. Rev. D 66 (2002) 015002 [hep-ph/0205001] [INSPIRE].

[55] J. Kang, P. Langacker, T.-j. Li and T. Liu, Electroweak baryogenesis in a supersymmetric U(1)-prime model, Phys. Rev. Lett. 94 (2005) 061801 [hep-ph/0402086] [INSPIRE]. 
[56] J. Erler, P. Langacker, S. Munir and E. Rojas, Improved Constraints on $Z^{\prime}$ Bosons from Electroweak Precision Data, JHEP 08 (2009) 017 [arXiv:0906.2435] [INSPIRE].

[57] J. Erler, P. Langacker, S. Munir and E. Rojas, Constraints on the mass and mixing of $Z^{\prime}$ bosons, AIP Conf. Proc. 1200 (2010) 790 [arXiv:0910.0269] [inSPIRE].

[58] F. del Aguila, J. de Blas and M. Pérez-Victoria, Electroweak Limits on General New Vector Bosons, JHEP 09 (2010) 033 [arXiv:1005.3998] [INSPIRE].

[59] J. Erler, P. Langacker, S. Munir and E. Rojas, Z' Searches: From Tevatron to LHC, arXiv: 1010.3097.

[60] Particle Data Group collaboration, K. Olive et al., Review of Particle Physics, Chin. Phys. C 38 (2014) 090001.

[61] ATLAS collaboration, Search for high-mass resonances decaying to dilepton final states in pp collisions at $\sqrt{s}=7 \mathrm{TeV}$ with the ATLAS detector, JHEP 11 (2012) 138 [arXiv: 1209.2535] [INSPIRE].

[62] R.K. Ellis, W.J. Stirling and B. Webber, QCD and collider physics, Camb. Monogr. Part. Phys. Nucl. Phys. Cosmol. 8 (1996) 1.

[63] J. Kang and P. Langacker, $Z$ ' discovery limits for supersymmetric $E_{6}$ models, Phys. Rev. D 71 (2005) 035014 [hep-ph/0412190] [INSPIRE].

[64] U. Baur, O. Brein, W. Hollik, C. Schappacher and D. Wackeroth, Electroweak radiative corrections to neutral current Drell-Yan processes at hadron colliders, Phys. Rev. D 65 (2002) 033007 [hep-ph/0108274] [INSPIRE].

[65] A.G. Dias, J.C. Montero and V. Pleitez, 3-3-1 models at electroweak scale, Phys. Lett. B 637 (2006) 85 [hep-ph/0511084] [INSPIRE]. 